\title{
Chemical zoning in minerals
}

\author{
Antao SM, Salvador JS, Cruickshank LA, Dhaliwal I \\ Department of Geoscience, University of Calgary, Calgary, Alberta T2N 1N4, Canada
}

Solid solutions are common in minerals, have a homogeneous composition, and are single phases that give rise to well-defined diffraction patterns. However, some minerals grow over a long period of geologic time (millions of years in some cases), so the growing conditions change. As a result, chemical compositions of such minerals are inhomogeneous. These minerals contain fine-scale chemical zoning that is similar to growth rings of a tree. The chemical composition of one zone is slightly different from another zone. There are usually a small number of such zones. Theoretically, each distinct composition should give rise to unique diffraction effects. Small compositional differences between phases can be detected by split diffraction peaks. The different compositions can be observed in back-scattered electron (BSE) maps. The Rietveld method and monochromatic short-wavelength synchrotron high-resolution powder X-ray diffraction (HRPXRD) data are used to characterize the different phases that are intergrown together. Some mineral examples will be discussed. 